\title{
Mathematical Modeling and Simulation based System Identification of Non-Minimum Phase Electro-Hydraulic Servo (EHS) System
}

\author{
Jahid Hasan, MS in ICE ${ }^{1}$, \\ Tanmoy Karmaker, BSc in EEE ${ }^{1}$, \\ Mazid Ishtique Ahmed, Associate Professor ${ }^{2}$ \\ ${ }^{1}$ Nanjing University of Posts and Telecommunications, China \\ ${ }^{1,2}$ Atish Dipankar University of Science and Technology,
}

\begin{abstract}
In this paper, Electro-Hydraulic Servo System (EHSS) is analyzed as an example of a physical non-minimum phase system. Mathematical modeling of the EHSS is presented by obtaining the transfer function of the detailed mechanical and electrical properties to classify in accordance to phase response. Obtained Pole-Zero Map (pzmap) through Simulation using MATLAB shows that the analyzed system has Non-minimum phase (NMP) behavior from the beginning. Step response simulation of the discussed system is done as further proof of systems NMP behavior. Effective control strategy to overcome the difficulties of closed loop control of EHA is also discussed. An adaptive control strategy is proposed after integrating a case study on different types of adaptive control method. Also a few estimation methods for the adaptation are studied.
\end{abstract}

Key words: TF, EHSS, pzmap, NMP, MRAC.

\section{INTRODUCTION}

From the history of modern control system, it can be stated that the most challenging factor faced by the control engineers in the solving process of any given system is the mathematical modeling. To establish control over a system, control engineers must primarily identify the type of that particular system. The possibility of having more than one input function and different units of variables creates the chance of system being unbalanced and harder to identify type of the system as a whole. The idea of summarizing all the different types of input variable together as a single unit paved the way to establish mathematical modeling.

So, modeling the system with mathematical functions as well as the designing of the full system is the primary task in the way to make a system controllable. Mathematical modeling makes the system analyzing task simpler by connecting the system with computerized control. According to Dutch control engineer Pieter Eykhoff modeling in system mathematics can be defined as "A representation of the essential aspects of an existing system (or a system to be constructed) which presents knowledge of that system in a usable form." Pieter Eykhoff also stated that"A mathematical model is an abstract model that uses mathematical language to describe the behavior of a system" [1].

System can be mainly classified in two types. One is Physical System and the other is Conceptual system. A physical system is comprised of matter and energy which exists in the real phenomenon. The objects of a physical system can be observed and experienced. On the other hand, a conceptual system is a non-physical system which comprised of values, abstract ideas or theories. The most significant concept of conceptual system is that by modeling the abstract system and estimating the probable output, the type of system can be foretold. The approaches of mathematical modeling of a system can be accomplished by using the following processes,

\section{Transfer Function \\ II. Differential Equation \\ III. State-Space Representation \\ IV.Statistical Model}

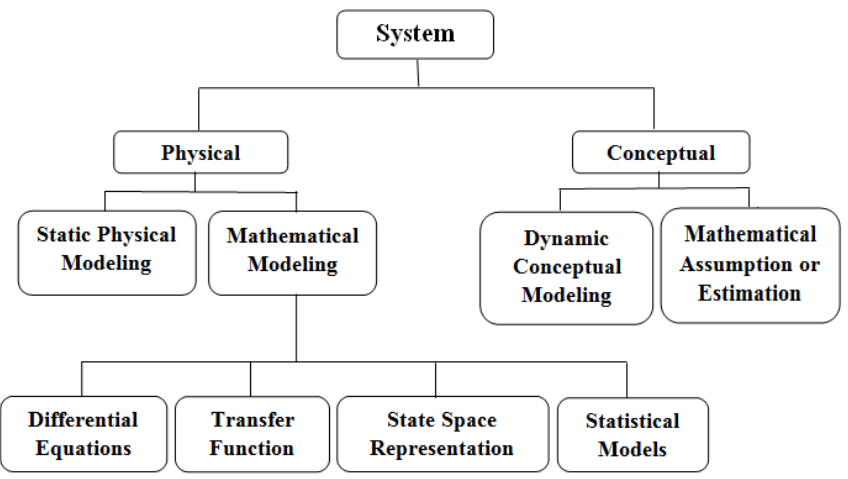

Fig. 1: Flow diagram of System Modeling.

Transfer function (TF) of a linear time invariant is the term which can be defined as the ratio of output function and input function when both the functions are converted in Laplace domain. Basically, Transfer Function creates a link relating the variables of systems inputs and outputs. The primary action of transfer function is to use the differential equation of the system input and output, then pinning them together under a single function. It does not depend upon the magnitude or the nature of the system but it provides significant ideas on the dynamic characteristics of the system [2].

Considering an LTI system defined using following differential equation,

$a_{0} y^{(n)}+a_{1} y^{(n-1)}+\ldots+a_{n-1} \dot{y}+a_{n}=b_{0} x^{(m)}+b_{1} x^{(m-1)}+\ldots+b_{m-1} \dot{x}+b_{m} x ;(n \geq m)$ 
Where, $\mathrm{x}$ is the input and $\mathrm{y}$ is the output for all possible values under $n \geq m$ condition. Now, transfer function is determined by taking Laplace Transform both side of the above equation. Transfer Function for the above system,

$$
\begin{gathered}
G(s)=\left.\frac{L[\text { Output }]}{L[\text { Input }]}\right|_{\text {zeroinitia lcondition } s} \\
G(s)=\frac{C(s)}{R(s)}=\frac{b_{0} s^{m}+b_{1} s^{m-1}+b_{2} s^{m-2}+\ldots+b_{m} s^{0}}{a_{0} s^{n}+a_{1} s^{n-1}+a_{2} s^{n-2}+\ldots+a_{n} s^{0}}
\end{gathered}
$$

Here, $C(s)$ is the Laplace Transform of the output function and $R(s)$ is the Laplace Transform of the input function and $G(s)$ is the transfer function under zero initial conditions.

In the control engineering system identification is the first and foremost task. Because of the presence of some systems with NMP Characteristics, controlling those systems properly becomes an aggravated challenge. To construct an effective control method for NMP system, first we have to study the identification methods of NMP system [3].

This research is about the discussion on the methods of System Identification and observing the behavior of the system. The prime topic that is being illustrated in this research is the behavior of a Non-minimum Phase (NMP) system. Our key motive was to find out the characteristics and phase behavior of a non-minimum phase system. We selected Electro-Hydraulic Servo System (EHSS) as the physical system. So, the purpose stood as to find and prove the NMP characteristics of EHSS from the step response and Pole-Zero Map using MATLAB. After identifying the nature of NMP, a suitable control strategy is proposed to minimize the instability and NMP characteristics from the EHSS.

\section{MATHEMATICAL FORMULATION}

\section{A. Non-minimum Phase System}

A non minimum phase system is a system which is inherently unstable because of having higher percentages of uncertainty. Non-minimum phase system has dead time problem on its transient response zone. In other words, when a system characteristic shows unstable and anti-causal conditions then the system can be called NMP systems. A given nonminimum phase system will have a greater phase contribution than the minimum-phase system with the equivalent magnitude response. It means that a NMP system is totally opposite of a MP system in contrast. As we discussed previously about the NMP system, the poles and zeros of a non minimum phase systems TF is in the right side of the unit circle for discrete-time systems.

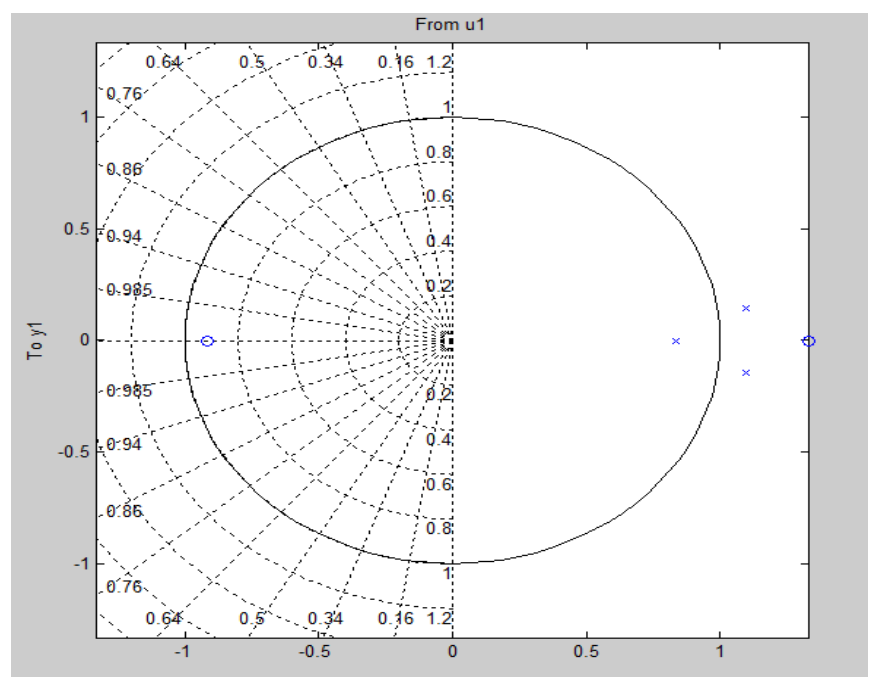

Fig. 2: Pole-Zero map of a random NMP System.

Figure 2 shows a Pole-Zero Map (pzmap) for a random non minimum phase system having discrete transfer function,

$$
\frac{Y(s)}{U(s)}=\frac{-0.003191 z^{2}+0.001324 z-0.003889}{z^{3}-3.028 z^{2}+3.053 z-1.02}
$$

In this pzmap, we can observe that there are two zeros and three poles present in it. Among these only one zero belongs to the left half plane (LHP) and the other poles and zeros can be identified on the right half plane (RHP) [4].

The transfer function of a Non-minimum phase system cannot be determined directly. It can only be estimated through the system properties and involved mechanical equation to a workable limit.

A non-minimum phase system can be classified in two types: One is Maximum Phase and another one is Mixed Phase system. Here, maximum phase system is totally opposite to the minimum phase system. A causal and stable LTI system is a maximum phase system if its inverse is causal and unstable. That means, poles and zeros are right side of the s-plane or outside of the unit circle. This maximum phase system has the maximum group delay.

\section{B. Difficulties faced in controlling NMP}

The basic problem with a non-minimum phase system is something called as internal stability. The response of a non minimum phase system to a step input has an "undershoot". This means, if the output was initially zero and the steady state output is positive, the output becomes first negative before changing direction and converging to its positive steady state value. This incident is annoying from a controller point of view. Imagine you take action to change the temperature of the water in your shower because it is too cold. However, 
before becoming warmer, the water becomes even colder. You may think in the first moment, you turned the knob in the wrong direction, so you turn it back. Well, this would be a wrong decision because this will make the water even colder in the long run. You may have noticed that this example is actually quite realistic in most shower systems. Or, for example a car driver wants to turn right. He would turn the power steering to the right. But imagine if the tires do not respond at the same time and takes a right turn after say a few seconds. This might result in a car clash or it may violate traffic rules which are not desirable at all.

\section{Electro-Hydraulic Servo System (EHSS)}

Electro-Hydraulic Servo system (EHSS) is a widely used electromechanical system which in general shows nonminimum phase properties. For this, we chose EHSS as the physical example of a non minimum phase system in this paper.

Hydraulic system is anelectro-mechanical system which operates with pressurized liquid flow. The working principle of Hydraulic system involves creating resistance or transmitting pressure of liquid when the pressurized liquid is passed forcefully through a small opening or tube. Hydraulic drive system is based on the Pascal's Law of pressure. It is a very commonly used actuating mechanism. Some best examples of Hydraulic system in present world are backhoes, cranes, excavator, and brake system used in vehicles.

Electro-Hydraulic system as the name implies, is a combinatory or a hybrid system which consists of both an electrical and a hydraulic system. This system is also an actuating process. Now, the question can come that what is the purpose of this system when we already have the Hydraulic system. The answer is simple- hydraulic system is dependent on movement of fluid through cylinders. In that case it is not fully safe to use this system for those purposes where the simplicity of architecture is needed. To overcome this limitation, the electro-hydraulic system is proposed.

The basic purpose of servo system is to provide motion control in a system. It consists of a disk made by semiconductor element. This disk's position is controlled precisely to control the movement of a system.

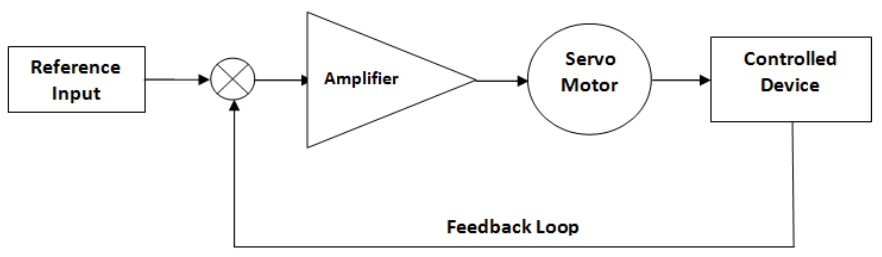

Fig. 3: Block Diagram of basic Servomechanism [5]
Figure 3 shows the basic block diagram of a servomechanism. In the figure we can see that the prime input is amplified by the amplifier and the amplified input then provided to the servo motor. The resultant then served into the controlled device.

Electro hydraulic Servo System (EHSS) is a closed loop control system, which is usually applied as an actuator unit to drive an object such as a rudder or vane. Depending on variable to be controlled, it can be a position, velocity or force control system. Electro hydraulic servo systems have the advantages of, precise and fine control, high power to weight ratio, small size, good load matching, high environmental stiffness, fast dynamic performances and wide adjustable speed range. Large inertia and torque loads can be handled with high accuracy and very rapid response. All these advantages are suitable for aerospace and missile applications[6].

\section{Mechanical Structure of EHSS}

In electric power transmission systems can classified as electrical or mechanical. Mechanical system can be classified into two types: One is hydraulic systems and another one is pneumatic systems.

The electro-hydraulic servo system which is the object of this study is composed of a hydraulic cylinder are driven by a servo valve which shown in figure 4 . In order to represent servo valve dynamics through a wider frequency range, a second order transfer function is used as approximation of the valve dynamics.

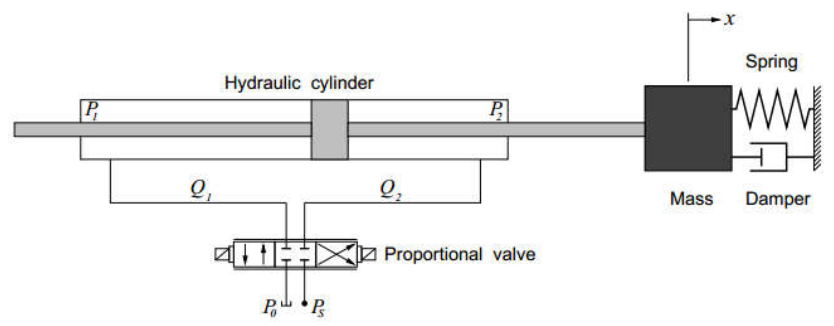

Fig. 4: Basic Schematic design of an EHSS. [7]

A hydraulic cylinder converts the energy stored in the hydraulic fluid into a force used to move the cylinder in a linear direction. A hydraulic cylinder consists of a cylindrical barrel, piston, and a piston rod. The piston that is placed within the barrel is connected to the piston rod.

The hydraulic fluid is an essential component of any hydraulic power or control system [8]. Hydraulic fluid depends on some physical system properties like temperature, viscosity and fluid stability, speed of response, lubricity and pressure. 
The dynamic equation of the EHSS system,

$$
\begin{gathered}
X p=V p \quad X p \\
m V_{p}=F_{a}-F f^{-d_{u}}
\end{gathered}
$$

Where,

$$
\begin{aligned}
& F_{a}=\text { Hydraulic Actuating Force } \\
& F_{f}=\text { Hydraulic Friction Force } \\
& \mathrm{m}=\text { mass of piston }
\end{aligned}
$$

\section{E. Mathematical Modeling of EHSS}

As it was stated before, mathematical modeling is essential to identify and control any system. By analyzing the mathematical model of a system, we can decide whether the system needs linearization or not and what kind of control approach we should adopt for that particular system.

Mathematical modeling of an EHSS means representing the hydraulic system components of EHSS in the form of equations. Mathematical modeling of the Electro hydraulic Servo System will be completed to make the system eligible for determining phase response and to find out the Pole-Zero Map. Mathematical modeling of an EHS System can be done neglecting the disturbances and the non-linearities of the system [9]. There is also the disturbance due to the dynamics of servo valve which is also can be disregarded.

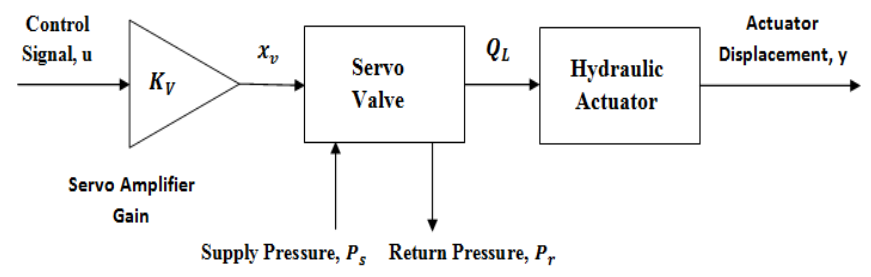

Fig. 5: Block Diagram of EHSS [9].

Figure 5 shows the block diagram of an Electro-Hydraulic Servo System. For determining the mathematical model of this system, firstly we have to neglect the dynamics of valve and the non-linear properties of the system. Thus, we can get an ideal system for modeling and estimation.

To connect the equations firstly we have to introduce the units that are involved in the EHSS system. Thus the units are shown below-

$u=$ Input Signal

$\mathrm{Xv}=$ Spool Valve Position

$\mathrm{Kv}=$ Servo Amplifier Gain

$\mathrm{QL}=$ Total Oil Load Flow

$\mathrm{Kq}=$ Flow Gain Coefficient

$\mathrm{Kc}=$ Flow Pressure Coefficient

$\mathrm{PL}=$ Load Pressure $\beta \mathrm{e}=$ Effective Bulk Modulus

$\mathrm{Vt}=$ Total Compressed Oil Volume

Ctp $=$ Total Leakage Coefficient

Ap $=$ Surface Area of the Piston

$\mathrm{y}=$ Actuator Displacement

$\mathrm{Fa}=$ Force of the Actuator

$\mathrm{Mt}=$ Total Mass of the Piston

$\zeta \mathrm{a}=$ Servo Valve Damping Coefficient

$\omega \mathrm{a}=$ Natural Frequency of the Actuator

Now, the relation of the servo amplifier gain $\boldsymbol{K}_{\boldsymbol{v}}$ and the spool valve position $\boldsymbol{X}_{\boldsymbol{v}}$ with the input signal $\boldsymbol{u}$ is given by the equation,

$$
X_{v}=K_{v} u
$$

This equation simply came from the gain theory of system where the gain is the ratio of input and output. Here, the input signal $\boldsymbol{u}$ can be either current or voltage.

Now, from a Tailor Series Linearization we get the equation of an EHA system dynamics for liquid (oil) flow $\boldsymbol{Q}_{L}$,

$$
Q_{L}=K_{q} X_{v}-K_{c} P_{L}
$$

The load pressure $\boldsymbol{P}_{\boldsymbol{L}}$ can be defined as the pressure across the servo actuator due to the flow of fluid $\boldsymbol{Q}_{\boldsymbol{L}}$ and the first derivative equation of the load pressure is given by the division of the total oil load flow through the hydraulic actuator $\boldsymbol{Q}_{\boldsymbol{L}}$ and the fluid capacitance. Now, the mathematical equation of first derivative of load pressure is given by,

$$
P_{L}=\frac{4 \beta_{e}}{V t}\left(Q_{L}-C_{t p} P_{L}-A P \dot{y}\right)
$$

After combining and substituting above equations we get,

$$
y=A_{p}^{2} \frac{4 \beta_{e}}{V_{t} M_{t}} \frac{K_{q} K_{v}}{A_{p}} u-\frac{4 \beta_{e}}{V_{t}}\left(K_{c}+C_{t p}\right) y-A_{p}^{2} \frac{4 \beta_{e}}{V_{t} M_{t}} y
$$

Now, moving the input signal into the Right hand side and the actuator displacement into the Left side of the above equation we get,

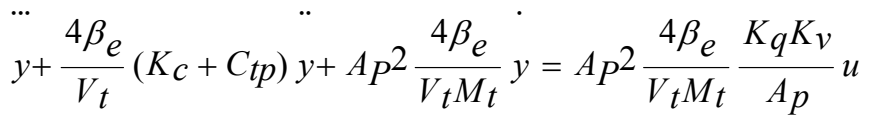

So the continuous time transfer function which can be determined from the above equation is,

$$
\frac{Y(s)}{U(s)}=\frac{A_{P} 2 \frac{4 \beta_{e}}{V_{t} M_{t}} \frac{K_{q} K_{v}}{A_{p}}}{s^{3}+s^{2} \frac{4 \beta_{e}}{V t}\left(K_{c}+C_{t p}\right)+s A_{P} 2 \frac{4 \beta_{e}}{V_{t} M_{t}}}
$$


Now, the transfer function determined by revising some of the constant values from equation 7 is,

$$
\frac{Y(s)}{U(s)}=\frac{\omega_{a}^{2} K_{a}}{s^{3}+2 \zeta_{a} \omega_{a} s^{2} \omega_{a}^{2 s}}
$$

Where,

Natural frequency, $\omega_{a}=A P \sqrt{\frac{4 \beta_{e}}{V_{t} M_{t}}}$

Total constant gain of the actuator, $K_{a}=\frac{K_{q} K_{v}}{A_{p}}$

And Damping coefficient of servo valve,

$$
\zeta_{a}=\frac{\sqrt{\frac{4 \beta_{e} M_{t}}{V_{t}}\left(K_{c}+C_{t p}\right)}}{2 A_{p}}
$$

Now, to find out the discrete time function from the continuous time function in equation 8 , we have to determine the function with zero order hold.The Zero-Order Hold $(\mathrm{ZOH})$ method provides an exact match between the continuous and discrete-time systems in the time domain for staircase inputs. The basic model of discrete time transfer function is,

$$
G(z)=\frac{y(k)}{u(k)}=\frac{b_{1} z^{2}+b_{2} z+b_{3}}{z^{3}+a_{1} z^{2}+a_{2} z+a_{3}}
$$

\section{SIMULATION AND ANALYSIS}

Basically a System can be Identified using Mathematical model. In order to understand the system behavior in easier way, MATLAB simulation on that mathematical model is adopted. As we described that an EHSS system inherits the uncertainties and it is a non-minimum phase system, the simulation result of an EHSS system will prove it.

\section{A. Simulation Data}

The values of parameters is collected from associated research work of M.I. AHMED \& A.K.M. AZAD [10] and they are being formed in a table shown below-

Table - 1: Adopted Parameters for Calculation

\begin{tabular}{|c|c|c|}
\hline $\begin{array}{c}\text { Name of the } \\
\text { Parameters }\end{array}$ & $\begin{array}{c}\text { Symbols of } \\
\text { parameters }\end{array}$ & Values \\
\hline Load Pressure & $P_{L}$ & $7 \mathrm{MPa}$ \\
\hline $\begin{array}{c}\text { Fluid mass } \\
\text { density }\end{array}$ & $\rho$ & $850 \mathrm{~kg} / \mathrm{m}^{3}$ \\
\hline
\end{tabular}

\begin{tabular}{|c|c|c|}
\hline $\begin{array}{c}\text { Surface area of } \\
\text { the piston }\end{array}$ & $A_{p}$ & $3 \times 10^{-4} \mathrm{~m}^{2}$ \\
\hline $\begin{array}{c}\text { Effective Bulk } \\
\text { modulus }\end{array}$ & $\beta_{e}$ & $700 \mathrm{MPa}$ \\
\hline $\begin{array}{c}\text { Total mass of the } \\
\text { piston }\end{array}$ & $M_{t}$ & $250 \mathrm{~kg}$ \\
\hline $\begin{array}{c}\text { Discharge } \\
\text { coefficient }\end{array}$ & $C_{d}$ & 0.6 \\
\hline $\begin{array}{c}\text { Natural frequency } \\
\text { of the actuator }\end{array}$ & $\boldsymbol{C}_{a}$ & $2.5 \times 10^{-2} \mathrm{~m}$ \\
\hline $\begin{array}{c}\text { Total leakage } \\
\text { coefficient }\end{array}$ & $C_{t p}$ & $2 \times 10^{-12} \mathrm{~m}^{3} / \mathrm{sPa}$ \\
\hline $\begin{array}{c}\text { Total } \\
\text { Compressed Oil } \\
\text { Volume }\end{array}$ & $V_{t}$ & $6 \times 10^{-5} \mathrm{~m}^{3}$ \\
\hline $\begin{array}{c}\text { Servo amplifier } \\
\text { gain }\end{array}$ & $K_{v}$ & $2.2 \times 10^{-6} \mathrm{~m} / \mathrm{V}$ \\
\hline $\begin{array}{c}\text { Flow Gain } \\
\text { Coefficient }\end{array}$ & $K_{q}$ & $1.8 \times 10^{-6} \mathrm{~m} / \mathrm{V}$ \\
\hline $\begin{array}{c}\text { Flow Pressure } \\
\text { Coefficient }\end{array}$ & $K_{c}$ & $75 \mathrm{~N} / \mathrm{m}$ \\
\hline
\end{tabular}

\section{B. Simulation using MATLAB}

To simulate the EHSS, we need the discrete time function of that system. Now, for finding the continuous time transfer function (Equation 8) after putting the values mentioned on table 1, we need to find the values of natural frequency, total constant gain of the actuator and damping coefficient of the servo valve at first.

So, Natural frequency,

$$
\omega_{a}=129.6148 \mathrm{rad} / \mathrm{sec}
$$

Constant gain of the actuator,

$$
K_{a}=1.320 \times 10^{-8}
$$

Damping Coefficient of Servo valve,

$$
\zeta_{a}=0.360
$$

Putting the values of $\omega_{a} K_{a}$ and $\zeta_{a}$ into equation 8 we get,

$$
\frac{Y(s)}{U(s)}=\frac{0.0002218}{s^{3}+93.33 s^{2}+1680 s}
$$

Now, determining discrete time transfer function from the above continuous transfer function by using continuous to discrete command with zero order hold in MATLAB. We get,

$$
\frac{Y(z)}{U(z)}=\frac{1.248 \times 10^{-9} z^{2}+5.055 \times 10^{-11} z-1.432 \times 10^{-13}}{z^{3}-1.017 z^{2}+0.01682 z-8.843 \times 10^{-5}}
$$




\section{Pole Zero Map Calculation}

Pole zero map is calculated to find the visual evidence that the Electro hydraulic servo system which is discussed in this paper is truly a non minimum phase system. The discrete transfer function of the EHSS is determined (Equation 10) at first and then the Pole zero map is found by using pzmap (sys) or pzplot (sys) command in the MATLAB. This gives us the Pole zero map which shows the number of poles (denominator polynomial roots) and zeros (numerator polynomial roots) of the transfer function and their location in the s-plane coordinates.

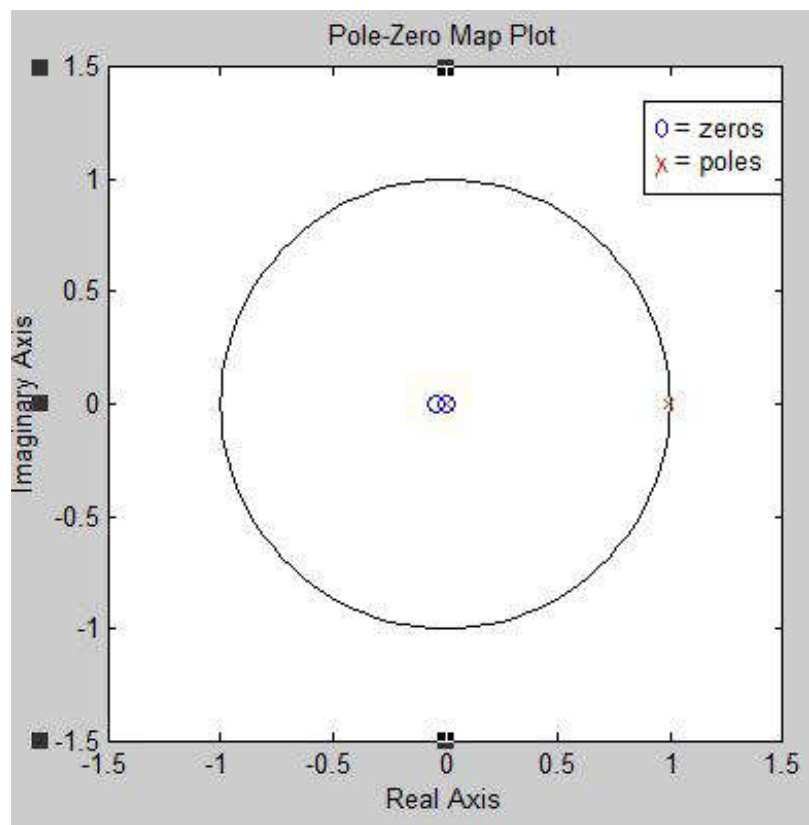

Fig. 6a: Pole Zero Plot of EHSS; without grid.

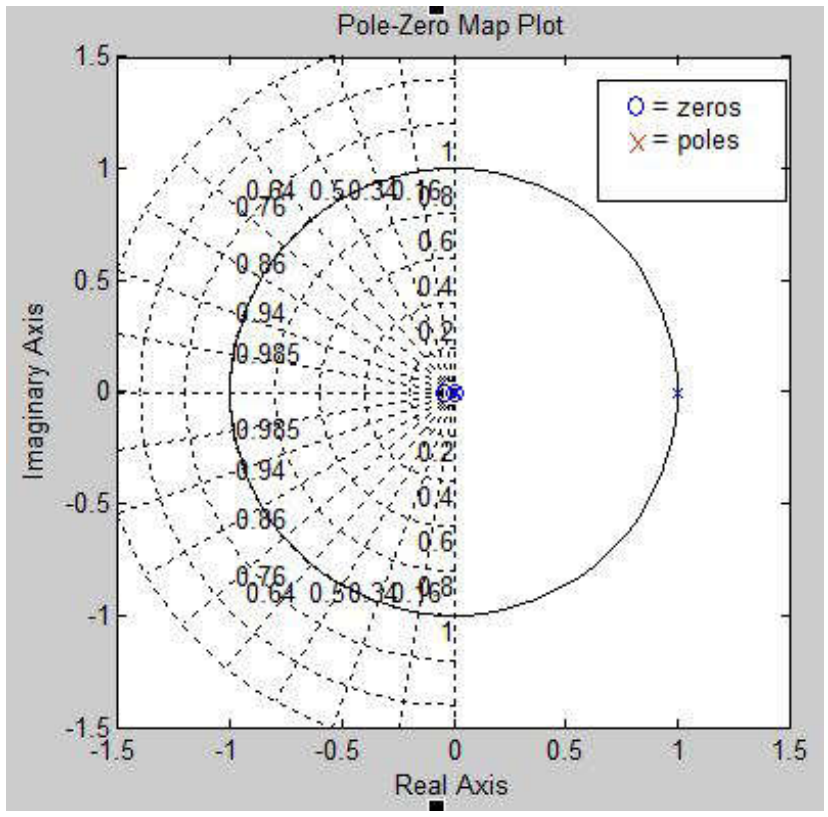

Fig. 6b: Pole Zero Plot of EHSS; with grid.
Here, in figure $6 a$ shows pole-zero map of the discrete time functions without the grid and figure $6 \mathrm{~b}$ shows the same polezero map with the grid on. The figure shows us that there are two zeros and three poles found in this system.

\section{Step Response of EHSS}

Step response of the EHSS system is determined next to find out the range of instability in the system due to uncertainty.By determining step response, we can find out two time frame at the same graph. One is the transient response or the constantly changing of amplitude against the change of time. The transient response is the step response beginning from the start to all the way before steady state point. The steady state response is the constant response following the settlement of transient response [11].

By measuring the step response we can analyze what happens before and after the step. This criterion is very helpful to relate with the estimation process that will be needed to control the non-minimum system.

Below, Fig 7 shows the steady state and the transient step output response with a high overshoot. This is the step response which is determined in MATLAB by using the discrete time transfer function of the EHSS (Equation 10).

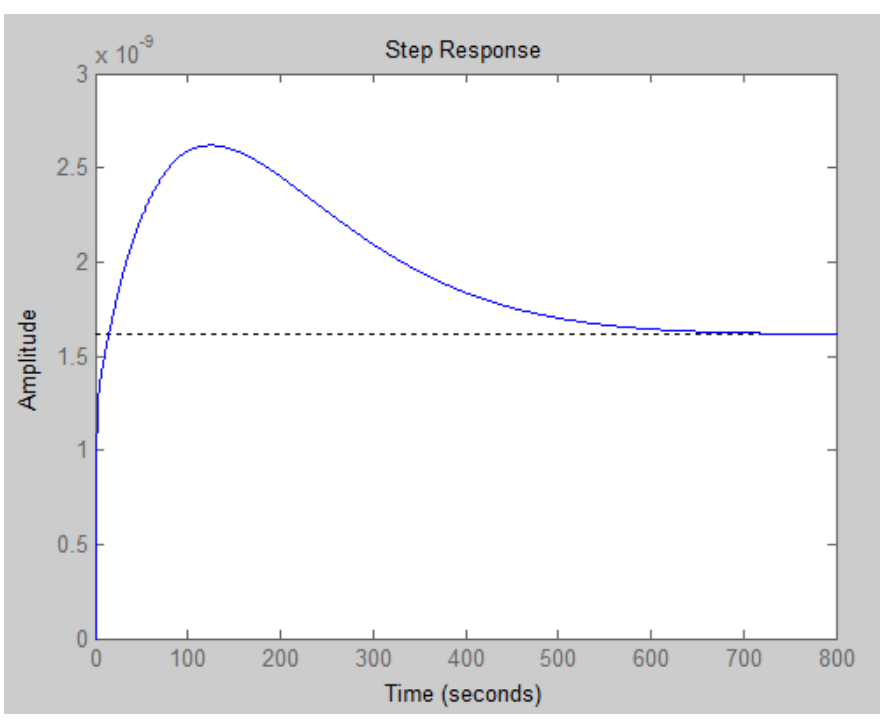

Fig. 7: Step Response of the EHSS

\section{E. Result Analysis of Simulation}

Now, from the result that we acquired from MATLAB simulation in section $\mathrm{C}$ and $\mathrm{D}$ we can see that the experimenting system is a Non-minimum phase system showing uncertainty and having dead time at the start. 


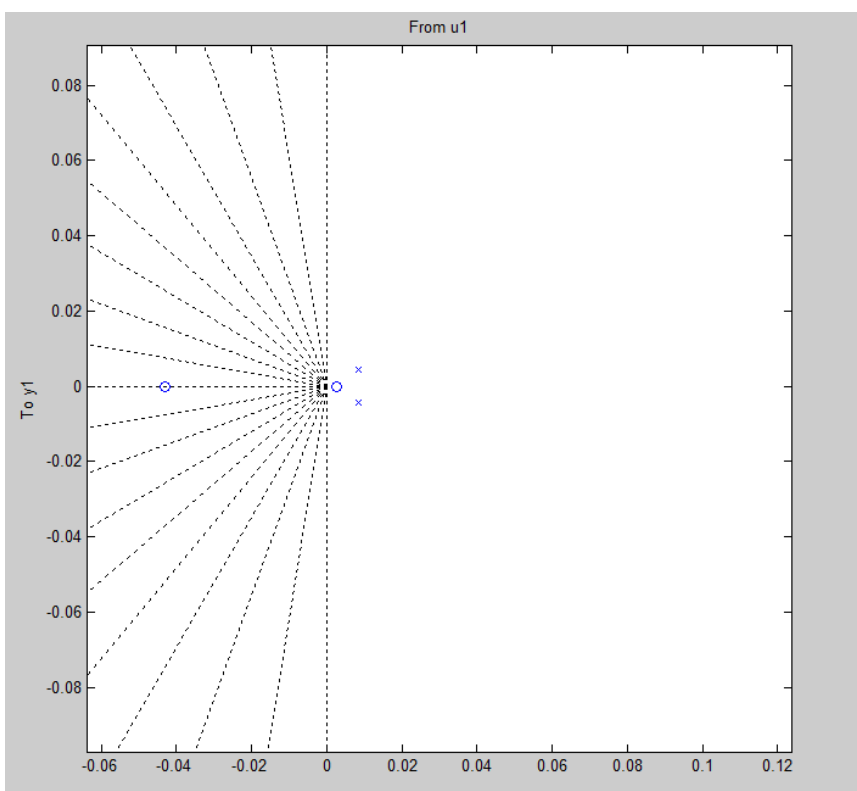

Fig. 8: Zoomed at the centre from figure $5 \mathrm{~b}$.

First of all, from the Pole-Zero map shown in figure $6 \mathrm{a}$ and $6 \mathrm{~b}$ we can see that there are two zeros and three poles in the system. For better viewing and understanding we can observe the zoomed version of figure $6 \mathrm{~b}$ which is shown in figure 8 . From the combination of both figure we can easily determine that only one zero is on the LHP and the other zero and three poles belongs to the RHP. As per the definition of Nonminimum phase system this is proven that the system is nonminimum phase and inherently unstable.

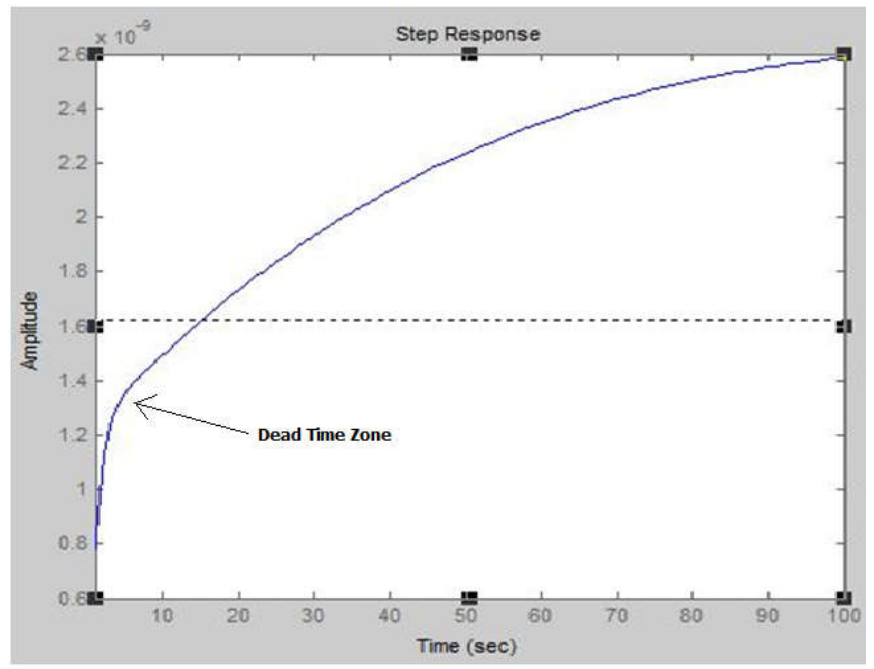

Fig. 9: Zoomed image of Step Response of EHSS

Up next is the step response of the system which is shown in figure $6 \mathrm{~b}$. Now, from the step response we can see that there is a gradual decrease in the amplitude against the rise of time before the normal steady state response is formed. That undershoot transient response is the dead time response which again proves that the system is non- minimum phase.

\section{CONCLUSSION}

The goal of this research work was to accumulate the theoretical and mathematical aspects on how a non minimum phase system is difficult to design. Also there was clear indication on the fact that a non minimum phase system is unstable hence the controlling of this types of system with conventional control method is proven to be obsolete. This paper also provides the physical examples of non-minimum phase system and the frequent uses of this type of system. This emphasizes on the importance of identifying a proper adaptive control method for this type of system. This research with further modification can be a base to implement the adaptation for real world system instruments.

So, it is easily understandable that the process of finding optimum control method and using it to complete the closed loop control for NMP system is vastly important. Also the system identification using mathematical modeling is equally important. At last, we believe that this research will help everyone to understand the importance of mathematical modeling and system identification for control system in the modern world.

For future work our target is to use the discussed estimation method to form an adaptive control strategy for the system [12], determine the closed loop transfer function, simulate the function to check whether the non-minimum phase properties is gone or not. And if the output comes as desired we will work on applying this method in a physical system. Then we plan to monitor its behavior and collect practical data to compare with the theoretical results.

\section{REFERENCES}

[1] Eykhoff P., " System identification: parameter and state estimation," Wiley,New York,1974, $555 \mathrm{p}$.

[2] Katsuhiko Ogata, "Modern Control Engineering” , 4th Edition.

[3] Lecture3, Discrete Time Signal Processing, OpenCourseWare-2006, Dept. of Electrical Engineering and Computer Science, MIT.

[4] Krishnendu Sarkar, "Controller Design for a Non-minimum Phase Electro-Hydraulic System”

[5] David Polka, “Motors \& Drives: A Practical Technology Guide” (c) 2003.

[6] Vijaya Sagar Tenali, "Simulation of Electro-hydraulic Servo Actuator."

[7] Wallace Moreira Bessa, Max Suell Dutra, and Edwin Kreuzer, "Adaptive Fuzzy Sliding Mode Control of Electro-hydraulic Servosystems”, DINAME 2009.

[8] A. Pamphlet, “ Engineering design handbook: Hydraulic fluids." Headquarters, US army material command, (C) 1971.

[9] Rozaimi Ghazali, Yahaya Md Sam, Mohd Fua' ad Rahmatand Zulfatman Has, “ Adaptive Discrete Sliding Mode Control for a Non Minimum Phase Electro Hydraulic Servo Actuator System " , Lecture Notes in Electrical Engineering, Springer, vol. 37, pp. 3-14, 2014.

[10]Mazid Istique Ahmed, A.K.M Azad, " Mathematical Modeling and DLQR based controller design for a non minimum phase Electrohydraulic servo system” , IEEE TENCON-2016.

[11]S. Salleh, M.F. Rahmat, S.M. Othman, “ Review on modeling and controller design of hydraulic actuator systems" , International Journal on Smart Sensing and Intelligent Systems, vol. 8(1), pp. 338-367, 2015.

[12] P. A. Ioannou, E.B. Kosmatopoulos, “Adaptive Control” , John Wiley \& Sons, Inc. (C) 1999. 